\title{
Relationship Between Preoperative Sarcopenia Status and Immuno-nutritional Parameters in Patients with Early-stage Non-small Cell Lung Cancer
}

\author{
FUMIHIRO SHOJI, TAICHI MATSUBARA, YUKA KOZUMA, NAOKI HARATAKE, \\ TAKAKI AKAMINE, SHINKICHI TAKAMORI, MASAKAZU KATSURA, \\ GOUJI TOYOKAWA, TATSURO OKAMOTO and YOSHIHIKO MAEHARA
}

Department of Surgery and Science, Graduate School of Medical Sciences, Kyushu University, Fukuoka, Japan

\begin{abstract}
Background: Although the skeletal muscle in the region of the third lumbar vertebra (L3) is generally assessed in order to judge sarcopenia, not every patient with non-small cell lung cancer (NSCLC) undergoes computed tomography including the $L 3$ region. We hypothesized that immunonutritional parameters could predict the existence of sarcopenia in patients with NSCLC. Aim: The aim of this study was to retrospectively investigate the correlation between preoperative sarcopenia and immuno-nutritional parameters in patients with early-stage NSCLC. Patients and Methods: We selected 147 of patients with pathological stage I NSCLC who underwent preoperative measurement of immuno-nutritional parameters and CT including the L3 region. Results: Preoperative sarcopenia was significantly associated with female gender $(p=0.0003)$ and poor prognosis $(p=0.0322)$. In Kaplan-Meier analysis of overall survival (OS) by preoperative sarcopenia status, the sarcopenic group had significantly shorter OS than the non-sarcopenic group (5-year OS: $87.27 \%$ vs. $77.37 \%, p=0.0131$, log-rank test). In multivariate analysis, the preoperative sarcopenia status (hazard ratio $=5.138$; 95\% confidence interval $=2.305-11.676$; $p<0.0001)$ was an independent prognostic factor. Preoperative sarcopenia status was significantly related to controlling nutritional status score $(p=0.0071)$ and Geriatric Nutritional Risk Index (GNRI) $(p<0.0001)$. Spearman's correlation test showed good significant correlation between preoperative
\end{abstract}

Correspondence to: Fumihiro Shoji, MD, Department of Surgery and Science, Graduate School of Medical Sciences, Kyushu University, 3-1-1 Maidashi, Higashi-ku, Fukuoka 812-8582, Japan. Tel: +81 926425466, Fax: +81 926425482, e-mail: fshoji@surg2.med.kyushu-u.ac.jp

Key Words: Non-small cell lung cancer, preoperative sarcopenia, immuno-nutritional parameters, Geriatric Nutritional Risk Index, predictor. sarcopenia status and GNRI ( $r=0.348, p<0.0001)$. Conclusion: The preoperative GNRI is a simple and useful predictor for existence of preoperative sarcopenia which was associated with poor outcome in patients with early-stage NSCLC.

Lung cancer is the leading cause of cancer-related death worldwide. The outcomes of early-stage lung cancer after curative surgical resection are still worse than for malignancies affecting other organs. Although adjuvant chemotherapy for patients with pathological stage IB and some with stage IA NSCLC has been established in Japan, the outcomes of patients with lung cancer is still not satisfactory. Thus, whether adjuvant therapy is performed and how the postoperative follow-up is planned are very important for patients with pathologically proven early-stage NSCLC in order to have survival benefits. Besides many prognostic factors, such as primary tumor characteristics, the nutritional status of patients with organ malignancies, including lung cancer, is an important topic.

Sarcopenia, which means depletion of skeletal muscle mass, was identified as a poor prognostic factor for organ malignancies such as pancreatic cancer, melanoma, hepatocellular carcinoma (1-3). In addition, we have previously reported that sarcopenia was also associated with poor outcome in male patients who underwent complete resection for early-stage NSCLC (4). However, there are some problems in evaluating sarcopenia in patients with NSCLC. Firstly, although the skeletal muscle in the region of the third lumbar vertebra (L3) is generally assessed in order to judge sarcopenia, not every patient with NSCLC undergoes CT including the L3 region. Secondly, it is necessary for judgment of sarcopenia to measure the skeletal muscle area, however, this requires much labor.

Immuno-nutritional parameters, including the Prognostic Nutritional Index (PNI), Controlling Nutritional Status (CONUT), and Geriatric Nutritional Risk Index (GNRI), have also been evaluated as predictive and prognostic factors 
of several malignancies. These parameters can be simply calculated based onhematological data and patient's anthropometric data. Recently, these immuno-nutritional parameters were identified as prognostic markers for several malignancies, including lung cancer (5-8).

Therefore, we hypothesized that immuno-nutritional parameters could predict the existence of sarcopenia in patients with NSCLC. Thus, the aim of this study was to retrospectively investigate the correlation between preoperative sarcopenia and immuno-nutritional parameters in patients with early-stage NSCLC.

\section{Materials and Methods}

Patients. Between August 2005 and August 2010. 391 consecutive patients with primary lung cancer underwent complete surgical resection at the Department of Surgery and Science, Graduate School of Medical Sciences, Kyushu University, Japan. For this study, we selected 147 of those patients with pathological stage I NSCLC who underwent preoperative measurement of immunonutritional parameters, including total lymphocyte count in the peripheral blood, serum albumin concentration, total cholesterol concentration and body weight. All patients underwent preoperative CT including the region at the third lumbar vertebra (L3). Images were analyzed using OsiriX, version 5.8 software (Pixmeo, Bernex, Switzerland). Sarcopenia assessment was performed from each patient's scan by evaluating a transverse CT image of the region at the L3 in the inferior direction.

The results were determined in follow-up examinations occurring over a median period of 59 (range=0-94) months after surgical resection. Postoperative follow-up consisted of chest and abdominal $\mathrm{CT}$, bone scintigraphy, and brain magnetic resonance imaging (MRI) at 6-month intervals during the first year and yearly thereafter. Chest radiograph and laboratory blood tests, including tumor markers were performed at 3- or 4-month intervals during the first year and at 6month intervals thereafter. Postoperative recurrence, such as local and distant recurrence, was defined as in a previous report (9). The first appearance of any new lesion suspected to be recurrence of the primary lung cancer lesion was defined as postoperative recurrence, and was clinically diagnosed by a combination of CT, MRI, bone scintigram, or fluorodeoxyglucose positron-emission tomography (FDG-PET), or it was pathologically diagnosed if necessary. Local recurrence was defined as that occurring in the hilar or mediastinal lymph nodes, pleural cavity, bronchial stump, or staple line. Lesions in other sites, including the brain, lung, adrenal glands, bone, and other locations were considered as distant recurrences. Clinically equivocal cases of second primary cancer were excluded in this study.

Calculation of skeletal muscle mass. Skeletal muscle was identified based on Hounsfield unit (HU) thresholds of -29 to +150 (water is defined as $0 \mathrm{HU}$, air as $1000 \mathrm{HU}$ ) (3). The following muscles in the L3 region were assessed: psoas, erector spinae, quadratus lumborum, transversus abdominis, external and internal oblique muscles, and the rectus abdominis (Figure 1). The cross-sectional areas $\left(\mathrm{cm}^{2}\right)$ of these muscles were computed automatically by adding together a given tissue's pixels and multiplying by pixel surface area in CT. Intraperitoneal organs and spinal cords were manually excluded. The cross-sectional areas were then normalized

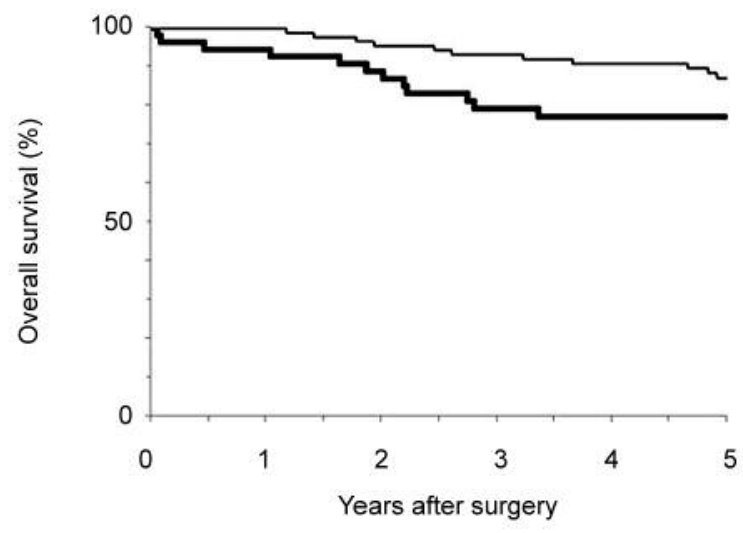

Patient at risk

No sarcopenia 90

Sarcopenia $\quad 57$

90

87

84

79

66

Figure 1. Kaplan-Meier curve analysis of overall survival (OS) for 147 patients with stage I non-small cell lung cancer by preoperative sarcopenia status. Bold line: Sarcopenic group; thin line: non-sarcopenic group. The sarcopenic group had a significantly shorter OS than the non sarcopenic group (5-year OS: $87.27 \%$ vs. $77.37 \%, p=0.0131$, log-rank test).

for height $\left(\mathrm{cm}^{2} / \mathrm{m}^{2}\right)$. Cut-off values for skeletal muscle were defined in accordance with a previous report as $43.75 \mathrm{~cm}^{2} / \mathrm{m}^{2}$ for men and $41.10 \mathrm{~cm}^{2} / \mathrm{m}^{2}$ for women (10). Based on this cut-off, patients were assigned to one of two groups, according to the presence or absence of sarcopenia. The clinicopathological background and rates of overall and recurrence-free survival of patients with and without sarcopenia were compared.

Preoperative calculation of immuno-nutritional parameters. Preoperative blood samples were obtained within 2 weeks before surgery. i) PNI. The preoperative PNI was calculated using the following formula: $10 \times$ serum albumin level $(\mathrm{g} / \mathrm{dl})+0.005 \times$ total lymphocyte count in peripheral blood (per $\left.\mathrm{mm}^{3}\right)(11)$.

ii) CONUT score. The preoperative CONUT score was calculated using serum albumin concentration, peripheral lymphocyte count, and total cholesterol concentrations (12). In brief, each parameter was scored as follows: Albumin concentration: $\geq 3.5 \mathrm{mg} / \mathrm{dl}$ : 0 points, 3.0-3.49 mg/dl: 2 points, $2.5-2.99 \mathrm{mg} / \mathrm{dl}: 4$ points, and $<2.5 \mathrm{mg} / \mathrm{dl}$ : 6 points. Total lymphocyte count: $\geq 1600 / \mathrm{mm}^{3}$ : 0 points, 1200 $1599 / \mathrm{mm}^{3}$ : 1 point, $800-1199 / \mathrm{mm}^{3}: 2$ points, and $<800 / \mathrm{mm}^{3}: 3$ points. Total cholesterol were scored as: $\geq 180 \mathrm{mg} / \mathrm{dl}$ : 0 points, $140-$ $179 / \mathrm{mm}^{3}: 1$ point, $100-139 / \mathrm{mm}^{3}: 2$ points, and $<100 / \mathrm{mm}^{3}: 3$ points. The sum of these scores was defined as the CONUT score.

iii) GNRI. The preoperative GNRI was calculated using serum albumin concentration and body weight as described elsewhere (5, 13). The GNRI formula is as follows: GNRI $=14.87 \times$ serum albumin concentration $(\mathrm{g} / \mathrm{l})+41.7 \times$ preoperative weight/ideal weight $(\mathrm{kg})$. The ideal body weight is calculated as follows: ideal body weight $=22 \times$ square of height $(\mathrm{m})$.

Histopathological evaluation. We retrospectively collected formalinfixed and paraffin-embedded NSCLC surgical specimens, stained them with hematoxylin and eosin, and reviewed the sections. Elastic 
Table I. Clinical profile of patients.

\begin{tabular}{lc}
\hline Characteristic & $\mathrm{N}=147$ \\
\hline Median follow-up (range), months & $59(0-94)$ \\
Median age (range), years & $68(42-86)$ \\
Gender, $\mathrm{n}(\%)$ & $65(44.2)$ \\
$\quad$ Female & $82(55.8)$ \\
$\quad$ Male & \\
Smoking status, $\mathrm{n}(\%)$ & $60(40.8)$ \\
$\quad$ Never & $87(59.2)$ \\
Former & $87(59.2)$ \\
Preoperative CEA level, $\mathrm{n}(\%)$ & $60(40.8)$ \\
$\quad$ Normal & $140(95.2)$ \\
$\quad$ Abnormal & $7(4.8)$ \\
Preoperative CYFRA level, $\mathrm{n}(\%)$ & $106(72.1)$ \\
$\quad$ Normal & $41(27.9)$ \\
Abnormal & \\
Surgical procedure, $\mathrm{n}(\%)$ & $19(12.9)$ \\
Lobectomy & $128(87.1)$ \\
$\quad$ Limited resection & \\
Adjuvant chemotherapy, $\mathrm{n}(\%)$ & \\
$\quad$ Yes & No
\end{tabular}

CEA, Carcinoembryonic antigen (normal value $<3.4 \mathrm{ng} / \mathrm{ml}$ ); CYFRA, cytokeratin 19 fragment (normal value $<3.5 \mathrm{ng} / \mathrm{ml}$ ).

and connective tissues were stained to determine pleural invasion, intratumoral blood vessel invasion (BVI), and lymphatic vessel invasion (LVI). BVI and LVI were distinguished by Elastica van Giessen staining. A specimen was considered positive for BVI when cancer cells were observed in the intratumoral vessel lumen. Pathological staging was based on the TNM classification of the International Union Against Cancer (14). For TNM staging, all of the patients underwent $\mathrm{CT}$ scans of the thorax and the upper abdomen, bone scintigrams, and brain CTs, MRIs, or FDG-PETs.

Outcome definition. Overall survival (OS) was defined as the time elapsed from resection to the date of death from any cause.

Statistical analysis. Clinical profiles of patients, pathological profiles of patients and patient characteristics were summarized using descriptive statistics or contingency tables. Fisher's exact test was used for analysis of categorical data. We analyzed patient survival using the Kaplan-Meier method and compared groups using the log-rank test. We used the Cox proportional hazards model to identify independent predictive and prognostic factors. The associations between preoperative sarcopenia and immunonutritional parameters were determined using Spearman's correlation tests. Values of $p<0.05$ were considered significant. All statistical analyses were performed using the JMP software program, version 11.0 (SAS Institute Inc., Cary, NC, USA).

\section{Results}

Clinical profiles. The patients' clinical profiles are shown in Table I. The study group included 65 women and 82 men, with a median age of 68 (range $=42-86)$ years at the time of
Table II. Pathological profile of patients $(N=147)$.

\begin{tabular}{lc}
\hline Characteristic & No (\%) \\
\hline Histological type & \\
Adenocarcinoma & $116(78.9)$ \\
Other & $31(21.1)$ \\
Tumor size & \\
T1a $(\leq 2 \mathrm{~cm})$ & $66(44.9)$ \\
T1b $(>2 \mathrm{~cm})$ & $34(23.1)$ \\
T2a & $47(32.0)$ \\
Pleural invasion (pl) & \\
pl 0 & $125(85.0)$ \\
pl 1-2 & $22(15.0)$ \\
Intratumoral blood vessel invasion & \\
No & $115(78.2)$ \\
Yes & $32(21.8)$ \\
Intratumoral lymphatic vessel invasion & $140(95.2)$ \\
No & $7(4.8)$ \\
Yes &
\end{tabular}

surgery. Sixty $(40.8 \%)$ patients had never smoked and the remaining patients were former or current smokers. Sixty $(40.8 \%)$ patients had abnormal preoperative carcinoembryonic antigen (CEA) levels (normal value: $<3.4 \mathrm{ng} / \mathrm{ml}$ ) and $7(4.8 \%)$ patients had abnormal preoperative cytokeratin 19 fragment (CYFRA) levels (normal value: $<3.5 \mathrm{ng} / \mathrm{ml}$ ). The majority of patients $(72.1 \%)$ underwent lobectomies with systemic lymphadenectomies and 41 patients underwent limited resections, including segmentectomies or wedge resections in those with peripheral lesions or poor pulmonary function. Nineteen (12.9\%) patients received adjuvant chemotherapy. Postoperative recurrence occurred in 24 patients (16.3\%).

Pathological profiles. The histological types were adenocarcinoma in $116(78.9 \%)$ patients and nonadenocarcinoma in $31(21.1 \%)$ patients. Of the 147 patients, $66(44.9 \%)$ had primary tumors $<2 \mathrm{~cm}$ (T1a). Twenty-two $(15.0 \%)$ patients were found to have visceral pleural invasion, 32 (21.8\%) had BVI, and seven (4.8\%) had LVI (Table II).

Correlation between preoperative sarcopenia status and clinic-pathological features. Preoperative sarcopenia was significantly associated with female gender $(p=0.0003)$ and poor prognosis $(p=0.0322)$, but with no other factors, including age, preoperative complications, histology, preoperative serum CEA and CYFRA levels, surgical procedures, pathological T factor, pleural invasion, BVI, or LVI (Table III).

Preoperative sarcopenia status and $O S$ in patients with pathological stage I NSCLC. In Kaplan-Meier analysis of OS by preoperative sarcopenia status, the sarcopenic group 
Table III. Clinicopathological characteristics in patients with and without sarcopenia. Data in the table are the number of patients in each category.

\begin{tabular}{|c|c|c|c|}
\hline Variable & $\begin{array}{c}\text { No sarcopenia } \\
\quad(n=90)\end{array}$ & $\begin{array}{l}\text { Sarcopenia } \\
\quad(\mathrm{n}=57)\end{array}$ & $p$-Value \\
\hline Age & & & 0.5480 \\
\hline$>75$ Years & 19 & 15 & \\
\hline$\leq 75$ Years & 71 & 42 & \\
\hline Gender & & & 0.0003 \\
\hline Male & 61 & 29 & \\
\hline Female & 29 & 36 & \\
\hline Preoperative complications & & & 0.4604 \\
\hline Yes & 11 & 11 & \\
\hline No & 41 & 27 & \\
\hline Histology & & & 0.6840 \\
\hline Non-adenocarcinoma & 18 & 13 & \\
\hline Adenocarcinoma & 72 & 44 & \\
\hline Preoperative CEA level & & & 0.8637 \\
\hline Abnormal & 36 & 24 & \\
\hline Normal & 54 & 33 & \\
\hline Preoperative CYFRA level & & & 0.7079 \\
\hline Abnormal & 5 & 2 & \\
\hline Normal & 85 & 56 & \\
\hline \multicolumn{4}{|l|}{ Surgical procedures } \\
\hline \multicolumn{4}{|l|}{$>0.9999$} \\
\hline Limited resections & 25 & 16 & \\
\hline Lobectomy & 65 & 41 & \\
\hline Pathological $\mathrm{T}$ factor & & & 0.2089 \\
\hline $\mathrm{T} 2 \mathrm{a}$ & 33 & 14 & \\
\hline $\mathrm{T} 1 \mathrm{ab}$ & 57 & 43 & \\
\hline Pleural invasion & & & 0.1935 \\
\hline pl 1-2 & 17 & 5 & \\
\hline pl 0 & 73 & 52 & \\
\hline \multicolumn{4}{|l|}{ Intratumoral blood } \\
\hline vessel invasion & & & 0.2186 \\
\hline Yes & 23 & 9 & \\
\hline No & 67 & 48 & \\
\hline \multicolumn{4}{|l|}{ Intratumoral lymphatic } \\
\hline vessel invasion & & & 0.7063 \\
\hline Yes & 5 & 2 & \\
\hline No & 85 & 55 & \\
\hline Postoperative recurrence & & & 0.2551 \\
\hline Yes & 12 & 12 & \\
\hline No & 78 & 45 & \\
\hline Outcome at end of follow-up & & & 0.0322 \\
\hline Dead & 12 & 16 & \\
\hline Alive & 78 & 41 & \\
\hline
\end{tabular}

CEA, Carcinoembryonic antigen (normal value $<3.4 \mathrm{ng} / \mathrm{ml}$ ); CYFRA, cytokeratin 19 fragment (normal value $<3.5 \mathrm{ng} / \mathrm{ml}$ ); GNRI, Geriatric Nutritional Risk Index.

had significantly shorter OS than the non-sarcopenic group (5-year OS=87.27\% vs. $77.37 \%$, respectively, $p=0.0131$, logrank test; Figure 1).

Prognostic factors in patients with pathological stage I NSCLC. We compared OS in patients by age (cut-off of 75 years), sex, smoking habit, preoperative serum CEA level, preoperative serum CYFRA level, preoperative sarcopenia status, pathological $\mathrm{T}$ factor, tumor type, extent of resection, BVI, LVI, and receipt of adjuvant (Table IV). Univariate analyses showed that age $(>75$ years $)(p<0.0001)$, male gender $(p=0.0009)$, with smoking history $(p=0.0243)$, abnormal preoperative serum CEA levels $(p=0.0003)$, preoperative sarcopenia $(p=0.0161)$, tumor size (T2a) ( $p=0.0003)$, with pleural invasion $(p=0.0007)$, and with BVI $(p=0.0150)$ significantly affected OS. In multivariate analysis, age ( $>75$ years) $(p=0.0006)$ and the preoperative sarcopenia existence $(p<0.0001)$ were independent prognostic factors (Table IV).

Correlation between preoperative sarcopenia status and immuno-nutritional parameters. Preoperative sarcopenia status was significantly negatively-associated with L3 skeletal muscle index $(p<0.0001)$, body mass index $(p<0.0001)$ and total lymphocyte count $(p=0.0074)$. According to immunonutritional parameters, preoperative sarcopenia status was significantly negatively-related to CONUT score $(p=0.0071)$ and GNRI $(p<0.0001)$, but not PNI $(p=0.1091)$ (Table V). Spearman's correlation test also showed significant correlation between preoperative sarcopenia status and both GNRI $(\mathrm{r}=0.348, \quad p<0.0001)$ and CONUT $(\mathrm{r}=-0.272$, $p<0.0001)$ but not PNI $(\mathrm{r}=0.174, p=0.0350)$.

\section{Discussion}

The present study retrospectively investigated the correlation between preoperative sarcopenia and immuno-nutritional parameters in patients with pathological stage I NSCLC. As a result, we show the preoperative sacrcopenia status was significantly correlated with preoperative immuno-nutritional parameters such as GNRI and CONUT score in surgically resected patients with pathological stage I NSCLC. Our result suggest that the preoperative immuno-nutritional parameters can be simplified to judge whether sarcopenia exists preoperatively not by measuring the skeletal muscle on CT but simply by calculating hematological data or using anthropometric measurement including body height and weight.

Sarcopenia, characterized by progressive loss of skeletal muscle mass, muscle strength and physical performance (15), has been recognized as clinically important. Sarcopenia can predict survival in patients with various kinds of cancer (13 ). Both cancer cachexia and sarcopenia are commonly observed in patients with advanced NSCL; several studies have reported sarcopenia was a poor prognostic factor in advanced NSCLC (16-19). However, few studies for patients with early-stage NSCLC have been reported. We previously reported that sarcopenia identified using a cross-sectional CT image of the L3 level was associated with poor outcome in patients with completely resected stage I NSCLC (4). 
Table IV. Univariate and multivariate analyses for overall survival in patients with stage I non-small cell lung cancer.

\begin{tabular}{|c|c|c|c|c|c|}
\hline \multirow[t]{2}{*}{ Variable } & \multirow[t]{2}{*}{ Comparison (comparator vs. Ref) } & \multicolumn{2}{|c|}{ Univariate analysis } & \multicolumn{2}{|c|}{ Multivariate analysis } \\
\hline & & $\mathrm{HR}(95 \% \mathrm{CI})$ & $p$-Value & $\operatorname{HR}(95 \% \mathrm{CI})$ & $p$-Value \\
\hline Age & $>75$ vs. $\leq 75$ Years & $5.875(2.745-12.764)$ & $<0.0001$ & $4.610(1.954-11.092)$ & 0.0006 \\
\hline Gender & Male $v s$. female & $4.207(1.733-12.531)$ & 0.0009 & $2.552(0.777-9.697)$ & 0.1260 \\
\hline Smoking status & Cur/for vs. never & $2.522(1.122-6.416)$ & 0.0243 & $1.571(0.531-5.122)$ & 0.4235 \\
\hline Preoperative CEA & Abnormal vs. normal & $4.125(1.880-9.975)$ & 0.0003 & $1.947(0.848-4.870)$ & 0.1183 \\
\hline Preoperative CYFRA & Abnormal vs. normal & $2.510(0.139-1.678)$ & 0.1818 & - & \\
\hline Preoperative sarcopenia & Yes vs. no & $2.499(1.187-5.406)$ & 0.0161 & $5.138(2.305-11.676)$ & $<0.0001$ \\
\hline Tumor size & $\mathrm{T} 2 \mathrm{a} v s . \mathrm{T} 1 \mathrm{ab}$ & $4.073(1.919-9.011)$ & 0.0003 & $2.097(0.725-5.824)$ & 0.1676 \\
\hline Histology & Non-Ad vs. Ad & $3.061(1.383-6.504)$ & 0.0068 & $1.470(0.591-3.526)$ & 0.3989 \\
\hline Procedure & Limited $v s$. lobectomy & $1.265(0.545-2.721)$ & 0.5675 & - & \\
\hline Pleural invasion & Yes vs. no & $4.410(1.941-9.478)$ & 0.0007 & $2.012(0.758-5.469)$ & 0.1594 \\
\hline BVI & Yes vs. no & $2.698(1.225-5.701)$ & 0.0150 & $1.396(0.599-3.127)$ & 0.4290 \\
\hline LVI & Yes $v s$. no & $1.643(0.265-5.503)$ & 0.5280 & - & \\
\hline Adjuvant chemotherapy & No vs. yes & $1.833(0.675-4.254)$ & 0.2157 & - & \\
\hline
\end{tabular}

HR, Hazard ratio; CI, confidence interval; Cur/for, current/former smoker; CEA, carcinoembryonic antigen (normal value $<3.4$ ng/ml); CYFRA, cytokeratin 19 fragment (normal value $<3.5 \mathrm{ng} / \mathrm{ml}$ ); limited, limited resection; Ad, adenocarcinoma; BVI, intratumoral blood vessel invasion; LVI, lymphatic vessel invasion; Ref, reference.

Table V. Preoperative immuno-nutritional parameters in patients with or without sarcopenia.

\begin{tabular}{lccc}
\hline Variable & Non-sarcopenia $(\mathrm{n}=90)$ & Sarcopenia $(\mathrm{n}=57)$ & $p$-Value \\
\hline L3 skeletal muscle index $\left(\mathrm{cm}^{2} / \mathrm{m}^{2}\right)$ & $50.5 \pm 6.8$ & $37.6 \pm 3.2$ & $<0.0001$ \\
Body mass index $\left(\mathrm{kg} / \mathrm{m}^{2}\right)$ & $23.7 \pm 2.8$ & $20.8 \pm 2.9$ & $<0.0001$ \\
Total lymphocyte count $\left(/ \mathrm{mm}^{3}\right)$ & $1849.5 \pm 588.9$ & $1581.3 \pm 611.4$ & 0.0074 \\
Albumin $(\mathrm{g} / \mathrm{dl})$ & $4.2 \pm 0.4$ & $4.2 \pm 0.3$ & 0.9369 \\
Total cholesterol $(\mathrm{mg} / \mathrm{dl})$ & $197.8 \pm 31.5$ & $194.1 \pm 32.8$ & 0.4936 \\
PNI & $51.1 \pm 4.9$ & $49.9 \pm 4.7$ & 0.1091 \\
CONUT & $0.8 \pm 1.1$ & $1.3 \pm 1.1$ & 0.0071 \\
GNRI & $107.4 \pm 8.3$ & $101.9 \pm 7.6$ & $<0.0001$
\end{tabular}

CONUT, Controlling nutritional status score; GNRI, geriatric nutritional risk index; PNI, prognostic nutritional index.

However, this relationship was found only in male not in female patients. We believe one reason why sex difference might have been found was that the sample size in that study was small $(n=90)$. Thus, in the present study, a total of 147 patients with pathological stage I NSCLC were assessed and significance of preoperative sarcopenia status was reevaluated. As a result, preoperative sarcopenia status was an independent prognostic factors in pathological stage I NSCLC regardless of sex. Preoperative sarcopenia status indeed has prognostic significance for patients surgically resected for early-stage NSCLC.

In addition, the present study also showed the significant correlation between sarcopenia and immuno-nutritional parameters in patients surgically resected for early-stage NSCLC. In particular, the preoperative GNRI had good discriminative power in predicting the existence of preoperative sarcopenia in patients with early-stage NSCLC in the present study. The GNRI has been reported to be a useful prognostic factor of esophageal cancer and renal cell cancer $(5,20)$. The GNRI reflects the nutritional status as it is based on serum albumin concentration and BMI. Serum albumin is a simple and valuable marker which can reflect malnutrition or cachexia in patients with cancer. Several studies have reported that body weight loss is considered a poor prognostic factor of lung cancer (21-24). Two studies evaluated patients that underwent lung cancer surgery and reported that preoperative BMI was a predictor of outcome after lung resection for lung cancer $(21,25)$. Thus, GNRI may be one of the most valuable immuno-nutritional parameters. An effective nutritional screening tool should be 
simple, costless and easy to use with readily available data. GNRI also can be easily calculated from routinely obtained laboratory data. Therefore, the existence of preoperative sarcopenia in early-stage NSCLC can be predicted by calculating the preoperative GNRI.

We previously reported that the PNI was a simple and novel predictor of postoperative recurrence in pathological stage I NSCLC (7). PNI was calculated using two parameters: the total lymphocyte count in peripheral blood and the serum albumin concentration (11). Thus, PNI can reflect both the immune response to cancer cells and the nutritional status. On the other hand, CONUT was calculated using three parameters including total lymphocyte count in peripheral blood, serum albumin concentrations and serum total cholesterol concentration (12). Low serum cholesterol levels are likely to be a secondary event after systemic inflammation (6). Therefore, the CONUT score enables evaluation of the protein reserve, calorie depletion, and immune defense. Consequently, the CONUT score can reflect immunological and nutritional status, and may be a good immuno-nutritional marker. In the present study, the preoperative CONUT score was also weakly associated with preoperative sarcopenia status, although there was no correlation between the preoperative PNI and sarcopenia status.

Jung et al. reported that sarcopenia was associated with an increased risk of grade 3-4 toxicity of chemotherapy and poor prognosis in patients with advanced colon cancer (26). Prado et al. studied the association between sarcopenia and adverse events in patients with breast cancer receiving chemotherapy (27). From these studies (26, 27), chemotherapy including induction or adjuvant therapy may be avoided for patients with organ malignancies and sarcopenia. In patients with advanced NSCLC receiving chemotherapy, sarcopenia is also associated with poor prognosis (18). Thus, sarcopenia seems to be a negative factor for chemotherapy in patients with advanced NSCLC.

However, there have been few studies of whether chemotherapy is beneficial or harmful for patients with preoperative sarcopenia with early-stage NSCLC. The outcomes after surgical resection in these patients are still not satisfactory compared with those with other organ malignancies, although patients with pathological stage IB or T1bN0M0 NSCLC have survival benefits from receiving adjuvant chemotherapy in Japan (7).

In the present study, we showed that preoperative sarcopenia status was an independent prognostic factor in patients with early-stage NSCLC. Therefore elucidating how patients with early-stage NSCLC with preoperative sarcopenia should be followed up or treated before and after surgical resection may further improve prognosis of patients with early-stage NSCLC. Therefore, the preoperative GNRI might be useful for selection of patients with early-stage
NSCLC needing intensive follow-up and induction or adjuvant therapy, including chemotherapy, radiotherapy or nutritional support.

However, this investigation has certain limitations. This study was a retrospective study from a single institution. Thus, a multicenter prospective study is necessary to evaluate the correlation between the preoperative sarcopenia status and GNRI and how to use preoperative GNRI for patient follow-up, and the survival benefit of therapy for patients with early-stage NSCLC with abnormal preoperative GNRI.

In conclusion, the preoperative GNRI is a simple and useful predictor for the existence of preoperative sarcopenia which was associated with poor outcome in patients with early-stage NSCLC.

\section{Conflicts of Interest}

None declared

\section{References}

1 Tan BH, Birdsell LA, Martin L, Baracos VE and Fearon KC: Sarcopenia in an overweight or obese patient is an adverse prognostic factor in pancreatic cancer. Clin Cancer Res 15: 6973-699, 2009.

2 Sabel MS, Lee J, Cai S, Englesbe MJ, Holcombe S and Wang S: Sarcopenia as a prognostic factor among patients with stage III melanoma. Ann Surg Oncol 18: 3579-3585, 2001.

3 Harimoto N, Shirabe K, Yamashita YI, Ikegami T, Yoshizumi T, Soejima Y, Ikeda T, Maehara Y, Nishie A and Yamanaka T: Sarcopenia as a predictor of prognosis in patients following hepatectomy for hepatocellular carcinoma. Br J Surg 100: 15231530, 2013

4 Suzuki Y, Okamoto T, Fujishita T, Katsura M, Akamine T, Takamori S, Morodomi Y, Tagawa T, Shoji F and Maehara Y: Clinical implications of sarcopenia in patients undergoing complete resection for early non-small cell lung cancer. Lung Cancer 101: 92-97, 2016.

5 Bo Y, Wang K, Liu Y, You J, Cui H, Zhu Y, Lu Q and Yuan L: The Geriatric Nutritional Risk Index predicts survival in elderly esophageal squamous cell carcinoma patients with radiotherapy. PLos One 11: e0155903, 2016.

6 Hirahara N, Matsubara T, Hayashi H, Takai K, Nakada S and Tajima Y: Prognostic importance of controlling nutritional status in patients undergoing curative thoracoscopic esophagectomy for esophageal cancer. Am J Ther, 2016. doi: 10.1097/MJT.00000 00000000414. [Epub ahead of print].

7 Shoji F, Morodomi Y, Akamine T, Takamori S, Katsura M, Takada K, Suzuki Y, Fujishita T, Okamoto T and Maehara Y: Predictive impact for postoperative recurrence using the preoperative prognostic nutritional index in pathological stage I non-small cell lung cancer. Lung Cancer 98: 15-21, 2016.

8 Osugi J, Muto S, Matsumura Y, Higuchi M, Suzuki H and Gotoh M: Prognostic impact of the high-sensitivity modified Glasgow prognostic score in patients with resectable non-small cell lung cancer. J Cancer Res Ther 12: 945-951, 2016. 
9 Varlotto JM, Recht A, Flickinger JC, Medford-Davis LN, Dyer AM and Decamp MM: Factors associated with local and distant recurrence and survival in patients with resected nonsmall cell lung cancer. Cancer 115: 1059-1069, 2009.

10 van Vledder MG, Levolger S, Ayez N, Verhoef C, Tran TC and Ijzermans JN: Body composition and outcome in patients undergoing resection of colorectal liver metastases. Br J Surg 99: 550-557, 2012.

11 Onodera T, Goseki N and Kosaki G: Prognostic nutritional index in gastrointestinal surgery of malnourished cancer patients. Nihon Geka Gakkai Zasshi 85: 1001-1005, 1984 (in Japanese).

12 Ignacio de Ulíbarri J, González-Madroño A, de Villar NG, González P, González B, Mancha A, Rodríguez F and Fernández G. CONUT: A tool for Controlling Nutritional Status. First validation in a hospital population. Nutr Hosp 20: 38-45, 2005.

13 Hasselmann M and Alix E: Tolls and procedures for screening for malnutrition and its associated in risks in hospital. Nutr Clin Metabol 17: 218-226, 2003.

14 Goldstraw P, Crowley J, Chansky K, Giroux DJ, Groome PA, Rami-Porta R, Postmus PE, Rusch V, Sobin L; International Association for the Study of Lung Cancer International Staging Committee; Participating Institutions: The IASLC lung cancer staging project: proposal for the revision of the TNM stage groupings in the forthcoming (seventh) edition of the TNM Classification of Malignant Tumours. J Thorac Oncol 2: 706714, 2007.

15 Cruz-Jentoft AJ, Baeyens JP, Bauer JM, Boirie Y, Cederholm T, Landi F, Martin FC, Michel JP, Rolland Y, Schneider SM, Topinková E, Vandewoude M, Zamboni M; European Working Group on Sarcopenia in Older People. Sarcopenia: European consensus on definition and diagnosis: report of the European Working Group on sarcopenia in older people. Age Aging 39: 412-423, 2010.

16 Baracos VE, Reiman T, Mourtzakis M, Gioulbasanis I and Antoun S: Body composition in patients with non-small cell lung cancer: a contemporary view of cancer cachexia with the use of computed tomography image analysis. Am J Clin Nutr 91: 1133S-1137S, 2010.

17 Martin L, Birdsell L, Macdonald N, Reiman T, Clandinin MT, McCargar LJ, Murphy R, Ghosh S, Sawyer MB and Baracos VE: Cancer cachexia in the age of obesity: skeletal muscle depletion is a powerful prognostic factor, independent of body masss index. J Clin Oncol 31: 1539-1547, 2013.

18 Kimura M, Naito T, Kenmotsu H, Taira T, Wakuda K, Oyakawa T, Hisamatsu Y, Tokito T, Imai H, Akamatsu H, Ono A, Kaira K, Murakami H, Endo M, Mori K, Takahashi T and Yamamoto N: Prognostic impact of cancer cachexia in patients with advanced non-small cell lung cancer. Support Care Cancer 23: 1699-1708, 2015 .
19 Sjøblom B, Grønberg BH, Wentzel-Larsen T and Baracos VE, Hjermstad MJ, Aass N, Bremnes RM, Fløtten $\varnothing$, Bye A and Jordh $\varnothing$ y M: Skeletal muscle radiodensity is prognostic for survival in patients with advanced non-small cell lung cancer. Clin Nutr 35: 1386-1393, 2016.

$20 \mathrm{Gu}$ W, Zhang G, Sun L, Ma Q, Cheng Y, Zhang H, Shi G, Zhu $\mathrm{Y}$ and Ye D: Nutritional screening is strongly associated with overall survival in patients treated with targeted agents for metastatic renal cell carcinoma. J Cachexia Sarcopenia Muscle 6: 222-230, 2015.

21 Nakagawa T, Toyazaki T, Chiba N, Ueda Y and Gotoh M: Prognostic value of body mass index and change in body weight in postoperative outcomes of lung cancer surgery. Interact Cardiovasc Thorac Surg 23: 560-566, 2016.

22 Yang R, Cheung MC, Pedroso FE, Byrne MM, Koniaris LG and Zimmers TA: Obesity and weight loss at presentation of lung cancer are associated with opposite effects on survival. J Surg Res 170: e75-83, 2011.

23 Topkan E, Parlak C and Selek U: Impact of weight change during the course of concurrent chemoradiation therapy on outcomes in stage IIIB non-small cell lung cancer patients: retrospective nalysis of 425 patients. Int J Radiat Oncol Biol Phys 87: 697-704, 2013.

24 Attaran S, McShane J, Whittle I, Poullis M and Shackcloth M: A propensity-matched comparison of survival after lung resection in patients with a high versus low body mass index. Eur J Cardiothorac Surg 42: 653-658, 2012.

25 Tan BH, Birdsell LA, Martin L, Baracos VE and Fearon KC: Sarcopenia in an overweight or obese patient is an adverse prognostic factor in pancreatic cancer. Clin Cancer Res 15: 6973-6979, 2009.

26 Jung HW, Kim JW, Kim JY, Kim SW, Yang HK, Lee JW, Lee KW, Kim DW, Kang SB, Kim KI, Kim CH and Kim JH: Effect of muscle mass on toxicity and survival in patients with colon cancer undergoing adjuvant chemotherapy. Support Care Cancer 23: 687-694, 2015.

27 Prado CM, Baracos VE, McCargar LJ, Reiman T, Mourtzakis M, Tonkin K, Mackey JR, Koski S, Pituskin E and Sawyer MB: Sarcopenia as a determinant of chemotherapy toxicity and time to tumor progression in metastatic breast cancer patients receiving capecitabine treatment. Clin Cancer Res 15: 2920-2926, 2009. 